Bull. Mater. Sci., Vol. 39, No. 6, October 2016, pp. 1581-1591. (c) Indian Academy of Sciences.

\title{
Structural, elastic, electronic and optical properties of bi-alkali antimonides
}

\author{
G MURTAZA $^{1, *}$ MAZHAR ULLAH ${ }^{1}$, NAEEM ULLAH ${ }^{2}$, MALIKA RANI ${ }^{3}$, M MUZAMMIL ${ }^{1}$, \\ R KHENATA $^{4}$, SHAHID M RAMAY ${ }^{5}$ and UMAIR KHAN ${ }^{6}$ \\ ${ }^{1}$ Materials Modeling Laboratory, Department of Physics, Islamia College University, Peshawar 25000, Pakistan \\ ${ }^{2}$ Department of Physics, G. D. C. Darra Adam Khel, F. R. Kohat, KPK 26000, Pakistan \\ ${ }^{3}$ Department of Physics, The Women University Multan, Multan 60000, Pakistan \\ ${ }^{4}$ LPQ3M Laboratory, Faculty of Science and Technology, University of Mascara, Mascara 29000, Algeria \\ ${ }^{5}$ Physics and Astronomy Department, College of Science, King Saud University, P.O. Box 2455, \\ Riyadh 11451, Saudi Arabia \\ ${ }^{6}$ Department of Physics, Hazara University, Mansehra, KPK 21300, Pakistan
}

MS received 7 September 2015; accepted 11 April 2016

\begin{abstract}
The structural parameters, elastic constants, electronic and optical properties of the bi-alkali antimonides ( $\mathrm{Na}_{2} \mathrm{KSb}, \mathrm{Na}_{2} \mathrm{RbSb}, \mathrm{Na}_{2} \mathrm{CsSb}, \mathrm{K}_{2} \mathrm{RbSb}, \mathrm{K}_{2} \mathrm{CsSb}$ and $\mathrm{Rb}_{2} \mathrm{CsSb}$ ) were calculated using state-of-the-art density functional theory. Different exchange-correlation potentials were adopted to predict the physical properties of these compounds. The calculated structural parameters are found in good agreement with the available experimental and theoretical results. All the compounds are mechanically stable. The compounds $\mathrm{Na}_{2} \mathrm{KSb}_{2} \mathrm{~K}_{2} \mathrm{RbSb}$, $\mathrm{K}_{2} \mathrm{CsSb}$ and $\mathrm{Rb}_{2} \mathrm{CsSb}$ have direct bandgaps, in which chemical bonding among the cations and anions is mainly ionic. Furthermore, the optical properties of these compounds are described in detail in terms of the dielectric function, refractive index, reflectivity, optical conductivity and absorption coefficient.
\end{abstract}

Keywords. Bi-alkali antimondies; elastic constants; electronic band structure; optical properties.

\section{Introduction}

The alkali and bi-alkali antimonides are highly quantum efficient semiconductors. These compounds are attracting interest of the research communities owing to their technological applications in photo-emissive and sensing devices $[1,2]$. The characteristics such as the photon absorption and feasible work function make these compounds suitable candidates for electron emission devices [3-5]. On the experimental side, high attention to alkali antimonides has been paid for exploration of the important electrical and optical properties for their use in technological applications [6-11].

The electronic structure calculations on the alkali antimonides have remained the central point of attention in the theoretical studies [12-19]. Ghosh [13] used the empirical pseudopotential method for calculating the band structure and the electronic properties of $\mathrm{Cs}_{3} \mathrm{Sb}$. The theoretical study on the structural stability of the alkali antimonides along with the intermetallic compounds have been done by Christensen [14] using the linear muffin tin orbital (LMTO) method. Using the full-potential linearized augmented plane wave (FP-LAPW) method, Wei and Zunger [15] explained the electronic structure in terms of the volume and the electrostatic interactions. The localized spherical wave method

\footnotetext{
*Author for correspondence (murtaza@icp.edu.pk)
}

has been used by Ettema and Groot [16-18] to study the electronic properties of alkali antimonides. The FP-LAPW method has been used by Ettema [19] and Kalarasse et al [20] for the electronic structure calculations of some alkali and bi-alkali antimonides.

Till date, mostly alkali antimonide compounds have been widely studied theoretically, while no comprehensive study has been devoted to examine the structural, elastic and optoelectronic properties of bi-alkali antimonide compounds regardless of their prospective technological applications. Motivated by the diverse technological applications, we have worked out the broad-range structural, elastic, electronic and optical properties of bi-alkali antimonides $\left(\mathrm{Na}_{2} \mathrm{KSb}\right.$, $\mathrm{Na}_{2} \mathrm{RbSb}, \mathrm{Na}_{2} \mathrm{CsSb}, \mathrm{K}_{2} \mathrm{RbSb}, \mathrm{K}_{2} \mathrm{CsSb}$ and $\mathrm{Rb}_{2} \mathrm{CsSb}$ ) using the full-potential linearized augmented plane wave plus local orbitals (FP-LAPW+lo) method within density functional theory [20].

\section{Computational details}

The present work was carried out in the framework of the FP-LAPW+lo method as implemented in the WIEN2k simulation package [20,21], which is one of the most accurate and efficient method for the calculation of the ground-state properties of materials [22,23]. In this method, the unit cell is divided into two regions; one region is occupied by the 
muffin-tin spheres, which contain the nuclei and core states, and the other part is the interstitial region containing the valence states. The plane wave cut-off $K_{\max }=10 / \mathrm{R}_{\mathrm{MT}}$ is used for the expansion of wavefunction in interstitial region, while $l_{\max }=10$ is used for spherical region. The exchangecorrelation potential was treated within the generalized gradient approximation (GGA) proposed by $\mathrm{Wu}$ and Cohen (WC-GGA) [24]. To overcome the bandgap underestimation of the WC-GGA predicted bandgap, Englo-Vosko GGA (EV-GGA) [25] and the modified version of Tran and Becke exchange potential [26] were used. The so-called (TB-mBJ potential) is also used for calculating the opto-electronic properties, which has previously shown close prediction to the experimental results [27]. An appropriate set of 1000 $\mathrm{k}$-points is used to ensure the energy convergence. Energy convergence threshold is taken as $0.001 \mathrm{eV}$ and the force convergence threshold is taken as $1 \mathrm{mRy}$ per a.u.

\section{Results and discussion}

\subsection{Structural properties}

The prediction of the structural parameters is necessary to account a material's structural behaviour. Here, the volume of the unit cell of each bi-alkali antimonide $\left(\mathrm{Na}_{2} \mathrm{KSb}\right.$, $\mathrm{Na}_{2} \mathrm{RbSb}, \mathrm{Na}_{2} \mathrm{CsSb}, \mathrm{K}_{2} \mathrm{RbSb}, \mathrm{K}_{2} \mathrm{CsSb}$ and $\mathrm{Rb}_{2} \mathrm{CsSb}$ ) compounds is optimized to obtain the structural parameters like lattice constant, $a(\AA)$, bulk modulus, $B(\mathrm{GPa})$, and its pressure derivative, $B^{\mathrm{p}}$. The optimized crystal structure of these bi-alkali compounds is shown in figure 1 . In the optimization procedure, the total energy of the unit cell for each compound is calculated by varying the unit cell volumes and plotted against corresponding energies using the Birch Murnaghan equation of state [28]. The optimization curves for the compounds are shown in figure 2. In the ground state, $a(\AA), B(\mathrm{GPa})$ and $B_{\mathrm{o}}$ are evaluated. The calculated values of these parameters are compared with the available theoretical and experimental results in table 1. The lattice constant $a(\AA)$ increases in one go from $\mathrm{Na}$ to $\mathrm{Rb}$. This increase in $a$ $(\AA)$ is attributed to the increase in atomic size of the anions from $\mathrm{Na}$ to $\mathrm{K}$ to $\mathrm{Rb}$ to $\mathrm{Cs}$. The bulk modulus $B(\mathrm{GPa})$ of the compounds is in the range of 12-21 GPa.

\subsection{Elastic properties}

The response of a material to the applied macroscopic stress can be described by calculating the elastic constants. For the cubic structure only three elastic constants are required to independently describe the mechanical properties. The elastic constants $C_{\mathrm{ij}}$ are estimated from first-principles calculations for the single crystals. For this purpose we have applied the Voigt-Reuss-Hill [29] approximation. In this technique, the actual effective modulus for polycrystals could be approximated by the arithmetic mean of the two wellknown bounds for monocrystals according to Voigt [30] and Reuss [31].

To predict $C_{11}$ and $C_{12}$, the first equation involves calculating the bulk modulus:

$$
B=\frac{1}{3}\left(C_{11}+2 C_{12}\right) \text {. }
$$

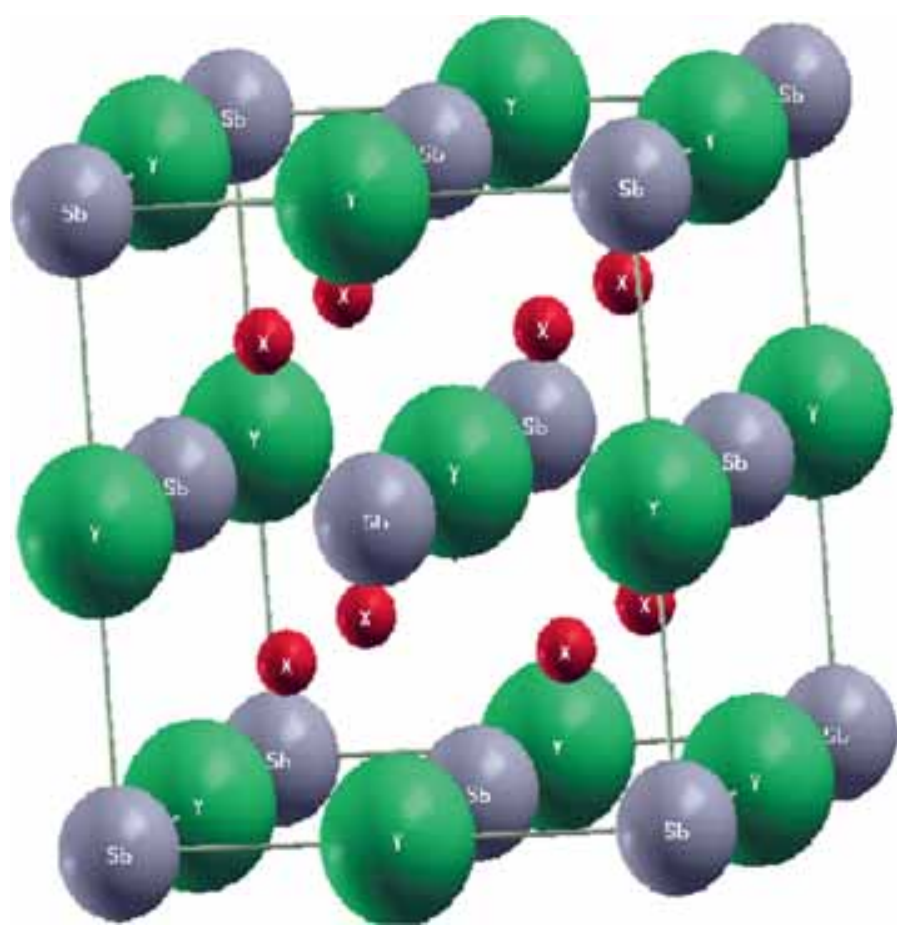

Figure 1. Crystal structure of the bi-alkali antimonides. 

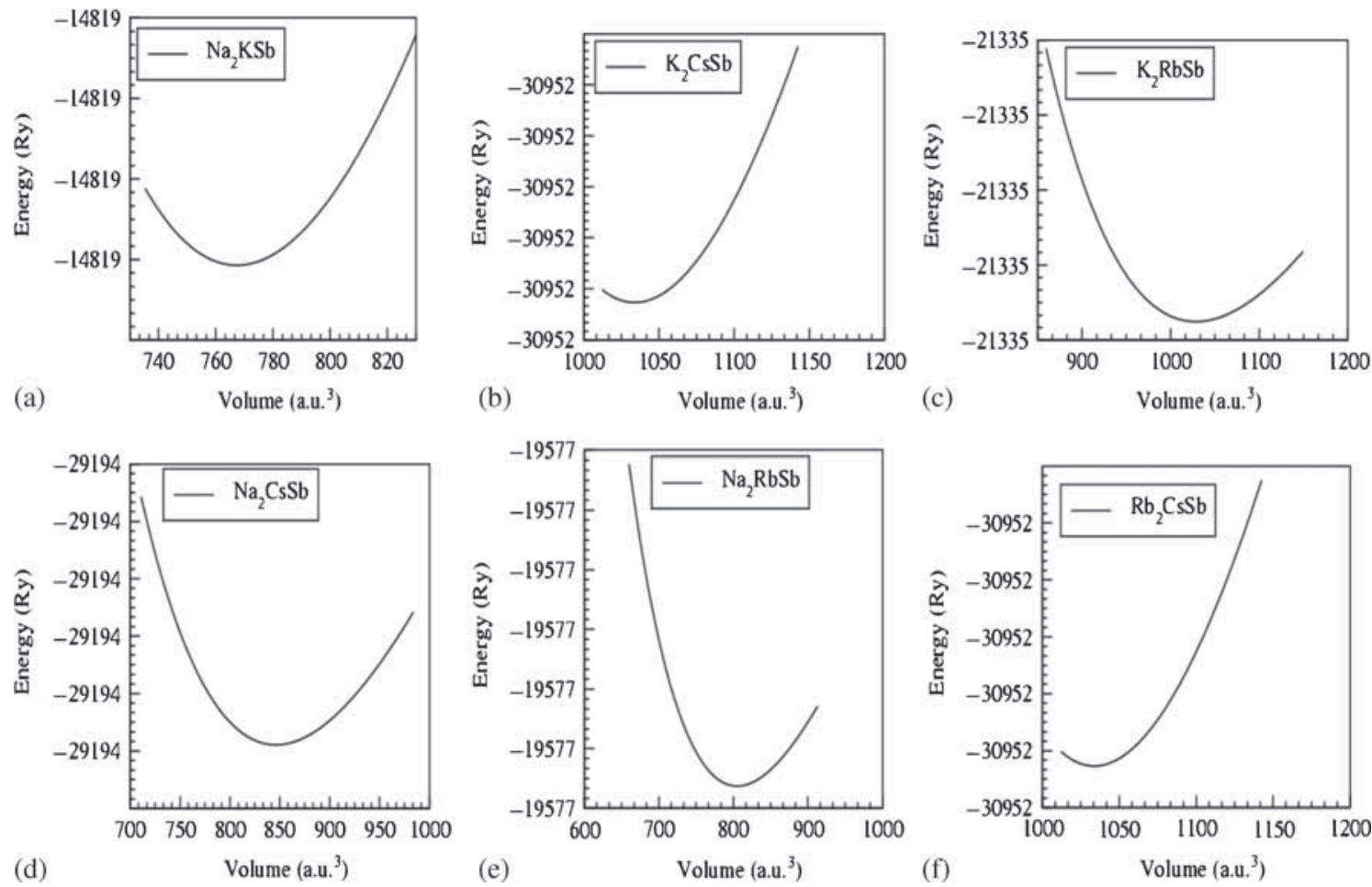

Figure 2. Optimization curve $(E-V)$ of the bi-alkali antimonides: (a) $\mathrm{Na}_{2} \mathrm{KSb}$, (b) $\mathrm{Na}_{2} \mathrm{RbSb}_{\text {, }}(\mathbf{c}) \mathrm{Na}_{2} \mathrm{CsSb}$, (d) $\mathrm{K}_{2} \mathrm{RbSb}$, (e) $\mathrm{K}_{2} \mathrm{CsSb}$ and (f) $\mathrm{Rb}_{2} \mathrm{CsSb}$.

Table 1. Optimized structural parameters of the bi-alkali compounds together with the experimental and theoretical data for comparison.

\begin{tabular}{lccccccc}
\hline & \multicolumn{3}{c}{ Lattice constant $(\AA)$} & & & & \\
Compounds & This work & Experimental & Others & $V_{0}(\text { a.u. })^{3}$ & $B(\mathrm{GPa})$ & $B^{\mathrm{P}}$ & $E_{0}(\mathrm{Ry})$ \\
\hline $\mathrm{Na}_{2} \mathrm{KSb}$ & 7.69 & $7.72^{\mathrm{a}}$ & $7.74^{\mathrm{b}}$ & 767.39 & 19.24 & 5.0 & -14818.56 \\
$\mathrm{Na}_{2} \mathrm{RbSb}$ & 7.81 & - & - & 805.13 & 18.64 & 6.42 & -19576.78 \\
$\mathrm{Na}_{2} \mathrm{CsSb}$ & 7.94 & - & - & 846.18 & 20.64 & 5.13 & -29193.84 \\
$\mathrm{~K}_{2} \mathrm{RbSb}$ & 8.47 & - & - & 1028.63 & 12.61 & 6.15 & -21334.99 \\
$\mathrm{~K}_{2} \mathrm{CsSb}$ & 8.56 & $8.61^{\mathrm{a}}$ & $8.508^{\mathrm{c}}$ & 1033.83 & 14.68 & 5.00 & -30952.07 \\
$\mathrm{Rb}_{2} \mathrm{CsSb}$ & 8.74 & - & - & 1128.6 & 13.76 & 9.62 & -40468.51 \\
\hline
\end{tabular}

${ }^{\mathrm{a}}$ Ref. [5], ${ }^{\mathrm{b}}$ Ref. [4], ${ }^{\mathrm{c}}$ Ref. [3], ${ }^{\mathrm{d}}$ Ref. [2].

Table 2. Calculated elastic constants and mechanical properties for bi-alkali hydrides.

\begin{tabular}{lrrrrrr}
\hline & $\mathrm{Na}_{2} \mathrm{KSb}$ & $\mathrm{Na}_{2} \mathrm{RbSb}$ & $\mathrm{Na}_{2} \mathrm{CsSb}$ & $\mathrm{K}_{2} \mathrm{RbSb}$ & $\mathrm{K}_{2} \mathrm{CsSb}$ & $\mathrm{Rb}_{2} \mathrm{CsSb}$ \\
\hline$C_{11}(\mathrm{GPa})$ & 22.28 & 77.91 & 30.91 & 16.15 & 38.24 & 18.00 \\
$C_{12}(\mathrm{GPa})$ & 19.39 & 23.57 & 14.25 & 11.87 & 3.13 & 10.87 \\
$C_{44}(\mathrm{GPa})$ & 17.59 & 31.25 & 8.95 & 15.06 & 23.29 & 13.01 \\
$B(\mathrm{GPa})$ & 20.35 & 41.68 & 19.80 & 13.30 & 14.83 & 13.25 \\
$C=C_{11}-C_{12}(\mathrm{GPa})$ & 6.31 & 7.77 & 0.25 & 19.50 & 3.65 & 1.70 \\
$G_{\mathrm{V}}(\mathrm{GPa})$ & 1.26 & 1.55 & 0.05 & 3.90 & 0.73 & 0.34 \\
$G_{\mathrm{R}}(\mathrm{GPa})$ & 3.21 & 29.47 & 8.69 & 4.41 & 20.61 & 6.31 \\
$\mathrm{G}=\left(G_{\mathrm{V}}+G_{\mathrm{R}}\right) / 2(\mathrm{GPa})$ & 7.17 & 29.54 & 8.69 & 7.15 & 20.79 & 7.77 \\
$\sigma$ & 0.34 & 0.21 & 0.31 & 0.27 & 0.022 & 0.25 \\
$E$ & 19.25 & 71.70 & 22.75 & 18.19 & 42.52 & 19.51 \\
$A$ & 12.18 & 1.15 & 1.07 & 7.04 & 1.33 & 3.65 \\
$B / G$ & 2.83 & 1.41 & 2.27 & 1.86 & 0.71 & 1.70 \\
\hline
\end{tabular}


The second equation comprises the volume conserving tetragonal strain tenser:

$$
\left[\begin{array}{ccc}
\delta & 0 & 0 \\
0 & \delta & 0 \\
0 & 0 & \frac{1}{(1+\delta)^{2}}-1
\end{array}\right],
$$

while the total energy has the following expression:

$$
E(\delta)=E(0)+3\left(C_{11}-C_{12}\right) V_{0} \delta^{2}+\mathrm{O}\left(\delta^{3}\right) .
$$

Here, $V_{0}$ is the volume of the unit cell.

To calculate $C_{44}$, the third equation contains the volume conserving rhombohedral strain tensor which is presented as follows:

$$
\frac{\delta}{3}\left[\begin{array}{lll}
1 & 1 & 1 \\
1 & 1 & 1 \\
1 & 1 & 1
\end{array}\right] .
$$
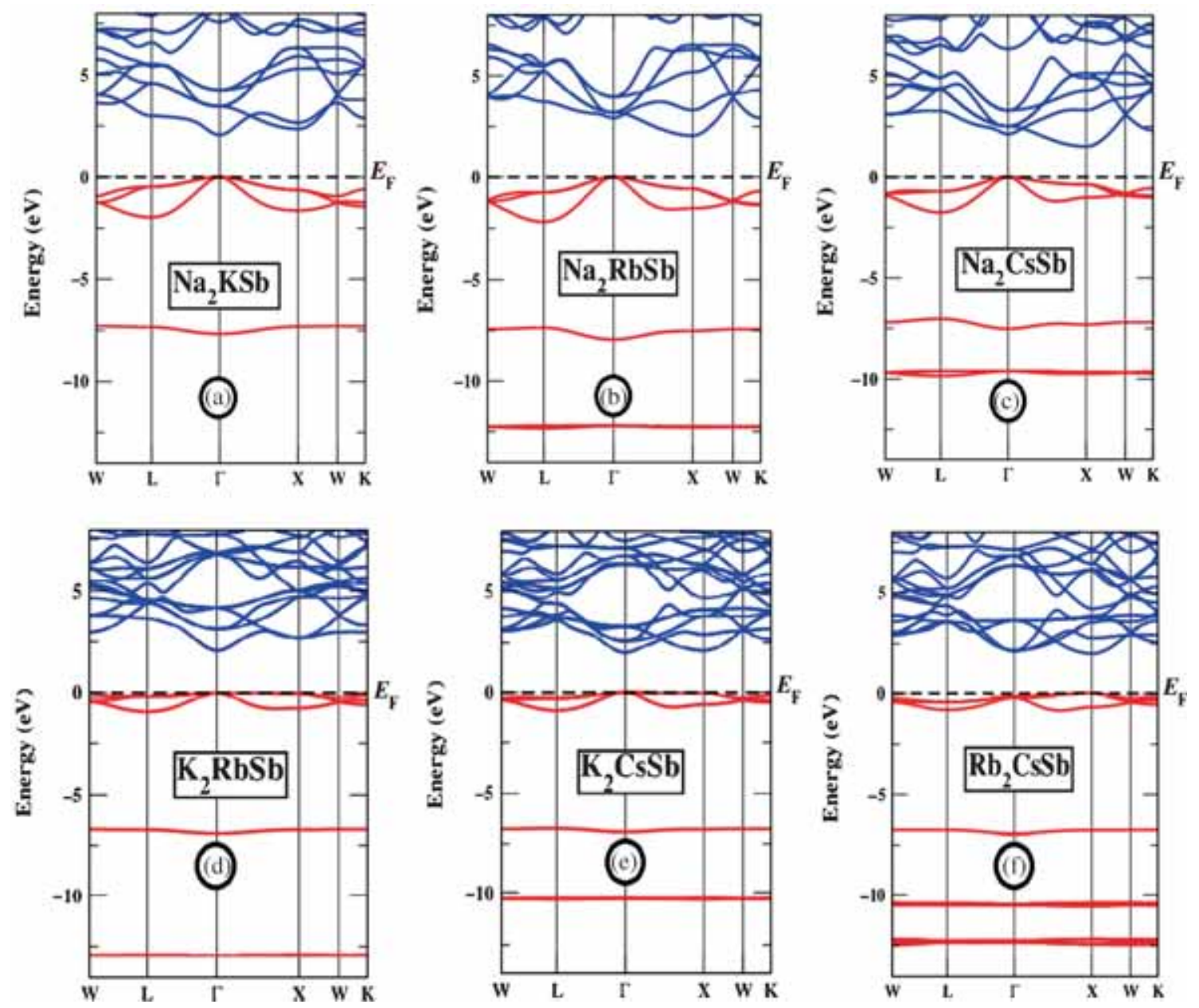

Figure 3. Calculated band structures of (a) $\mathrm{Na}_{2} \mathrm{KSb}$, (b) $\mathrm{Na}_{2} \mathrm{RbSb}$, (c) $\mathrm{Na}_{2} \mathrm{CsSb}$, (d) $\mathrm{K}_{2} \mathrm{RbSb}$, (e) $\mathrm{K}_{2} \mathrm{CsSb}$ and (f) $\mathrm{Rb}_{2} \mathrm{CsSb}$.

\begin{tabular}{|c|c|c|c|c|c|c|}
\hline \multirow[b]{2}{*}{ Compound } & \multicolumn{3}{|c|}{$E_{\mathrm{g}}(\mathrm{eV})$} & \multirow[b]{2}{*}{ Nature } & \multirow[b]{2}{*}{ Others } & \multirow[b]{2}{*}{ Experimental } \\
\hline & WC-GGA [24] & EV-GGA [25] & TB-mBJ [26] & & & \\
\hline $\mathrm{Na}_{2} \mathrm{KSb}$ & 0.56 & 1.22 & 2.06 & Direct & - & $1.1^{\mathrm{a}}$ \\
\hline $\mathrm{Na}_{2} \mathrm{RbSb}$ & 1.13 & 1.61 & 2.04 & Indirect & - & - \\
\hline $\mathrm{Na}_{2} \mathrm{CsSb}$ & 0.64 & 1.10 & 1.50 & Indirect & - & - \\
\hline $\mathrm{K}_{2} \mathrm{RbSb}$ & 0.54 & 1.21 & 2.08 & Direct & - & - \\
\hline $\mathrm{K}_{2} \mathrm{CsSb}$ & 0.80 & 1.58 & 1.99 & Direct & $1.13^{\mathrm{b}}$ & $1.2^{\mathrm{a}}$ \\
\hline $\mathrm{Rb}_{2} \mathrm{CsSb}$ & 1.21 & 1.78 & 1.98 & Direct & - & - \\
\hline
\end{tabular}

Table 3. Energy bandgaps of bi-alkali antimonides calculated with the TB-mBJ, EV-GGA and WC-GGA approximations.

${ }^{\mathrm{a}}$ Ref. [7], ${ }^{\mathrm{b}}$ Ref. [17]. 
The total energy then expressed as a function of deformation:

$$
E(\delta)=E(0)+\frac{1}{6}\left(C_{11}+2 C_{12}+4 C_{44}\right) V_{0} \delta^{2}+\mathrm{O}\left(\delta^{3}\right) .
$$

In this way the moduli for the cubic structure are defined as:

$$
G_{\mathrm{v}}=\frac{1}{5}\left(C_{11}-C_{12}+3 C_{44}\right), \quad \text { (6) } \quad G=\frac{G_{\mathrm{v}}+G_{\mathrm{R}}}{2} .
$$

$G_{\mathrm{R}}=\frac{5 C_{44}\left(C_{11}-C_{12}\right)}{4 C_{44}+3\left(C_{11}-C_{12}\right)}$.

The shear modulus $G$ is given by:

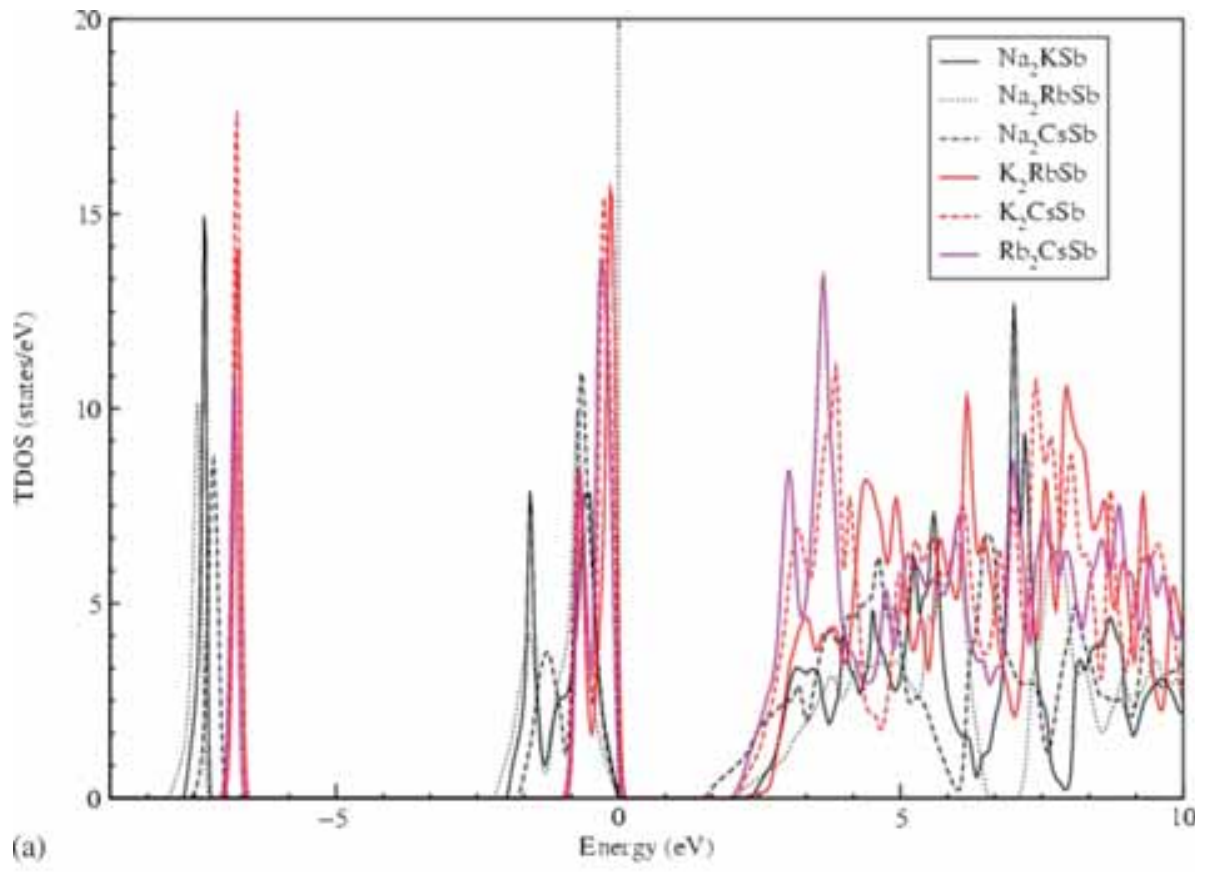

(a)

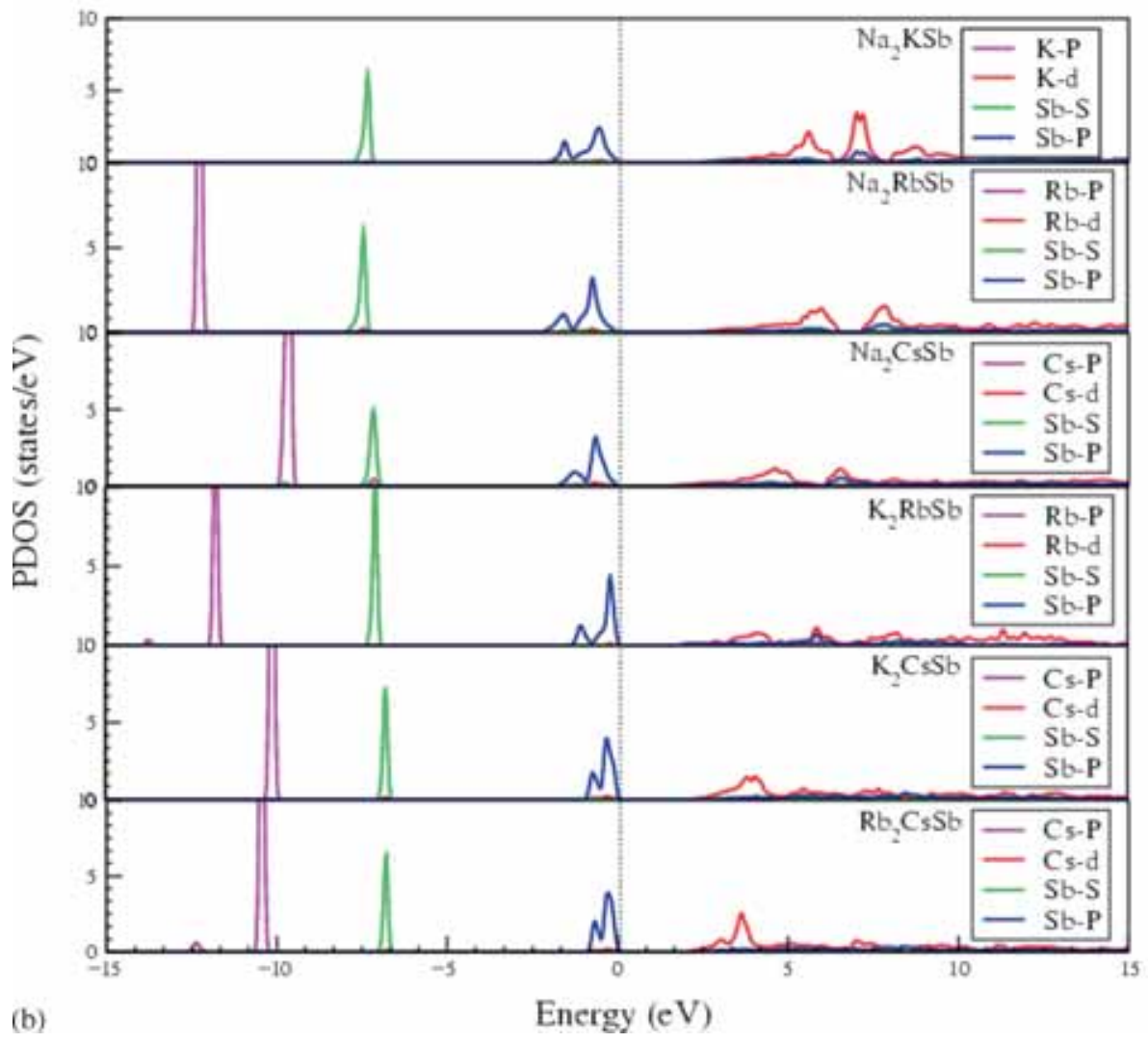

Figure 4. (a) Total density of state (TDOS) and (b) partial density of state (PDOS) of the bi-alkali antimonides. 


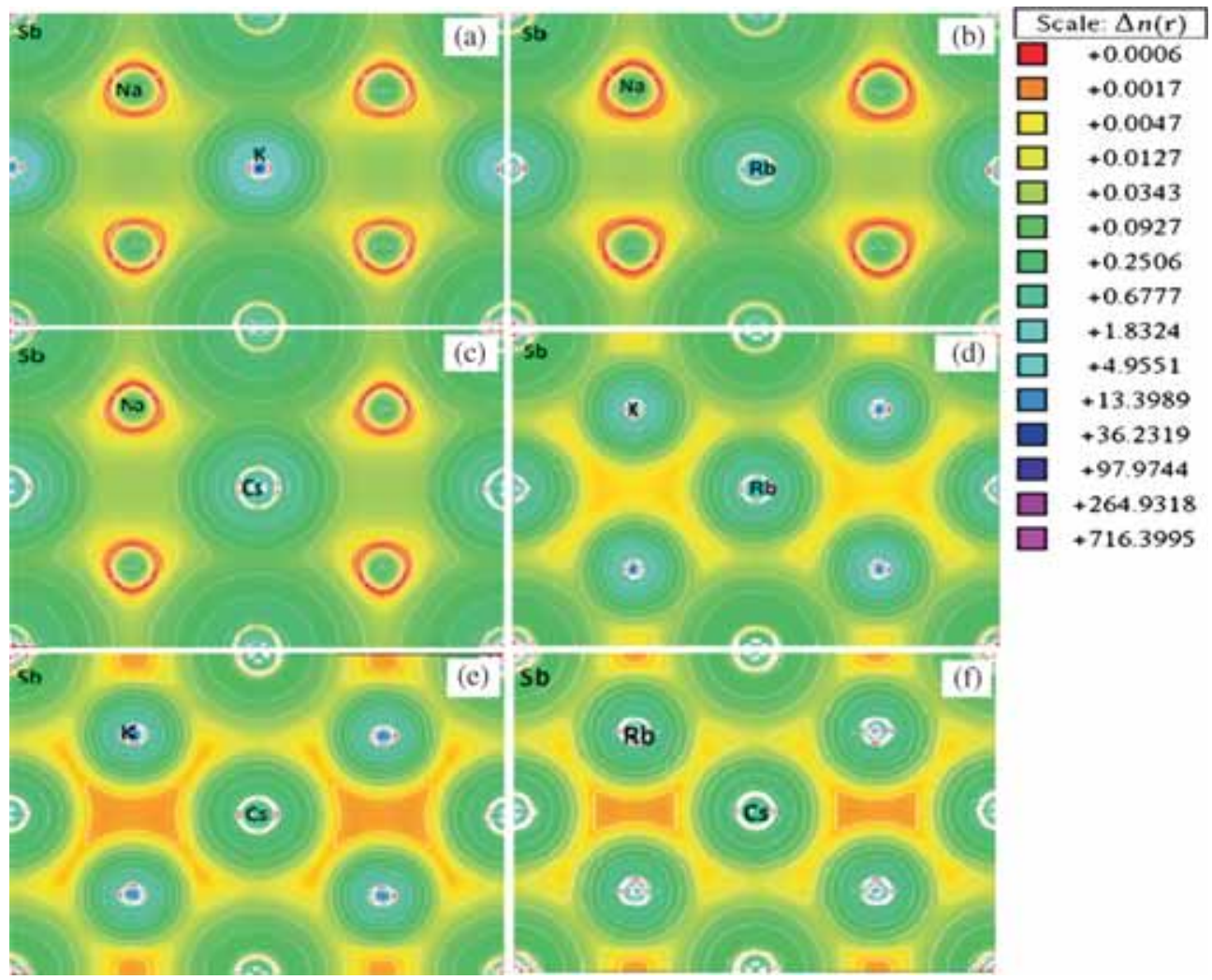

Figure 5. Electron charge density difference plots of the bi-alkali antimonides.

We have also predicted Young's modulus, E, and the Poisson's ratio, $v$, which are related to the bulk modulus $B$ and the shear modulus $G$ by the following equations [32,33]:

$$
\begin{aligned}
& E=\frac{9 B G}{(3 B+G)}, \\
& \nu=\frac{(3 B-2 G)}{2(3 B+G)} .
\end{aligned}
$$

The anisotropy factor, $A$, of materials is defined by the following expression:

$$
A=\frac{2 C_{44}}{\left(C_{11}-C_{12}\right)} \text {. }
$$

Table 2 summarizes the calculated elastic constant along with the important mechanical properties. There are no previous experimental or other theoretical data to compare with and therefore can serve as reference for further examinations on these compounds. The important elastic properties for the compounds have been calculated. All the compounds are elastically stable. The bulk modulus of $41.67 \mathrm{GPa}$, shear modulus of $29.55 \mathrm{GPa}$ and Young's modulus of $71.70 \mathrm{GPa}$ are highest for $\mathrm{Na}_{2} \mathrm{RbSb}$. Anisotropy factor for $\mathrm{Na}_{2} \mathrm{CsSb}$ is 1.07, which shows its isotropic nature, while for the remaining compounds this factor deviates from the critical value of unity (1.0). Pugh's index of ductility [34] for $\mathrm{Na}_{2} \mathrm{KSb}$, $\mathrm{Na}_{2} \mathrm{CsSb}$ and $\mathrm{K}_{2} \mathrm{RbSb}$ is more than the critical value 1.75 , reflecting their ductile nature, while the others are brittle.
Poisson's ratio of $\mathrm{Na}_{2} \mathrm{KSb}, \mathrm{Na}_{2} \mathrm{CsSb}$ and $\mathrm{K}_{2} \mathrm{RbSb}$ is greater than 0.26 , which means that these compounds are ductile.

\subsection{Electronic properties}

The calculated band structures along high symmetry points inside first Brillouin zone using TB-mBJ approximation for the studied compounds are displayed in figure $3 \mathrm{a}-\mathrm{f}$. One can see from these figures that $\mathrm{Na}_{2} \mathrm{RbSb}$ (b) and $\mathrm{Na}_{2} \mathrm{CsSb}$ (c) are indirect bandgap compounds, while the others are direct bandgap materials. Figure 3 illustrates the direct $(\Gamma-\Gamma)$ bandgap in $\mathrm{Na}_{2} \mathrm{KSb}, \mathrm{K}_{2} \mathrm{RbSb}$ and $\mathrm{K}_{2} \mathrm{CsSb}$ materials, while the direct bandgap for $\mathrm{Rb}_{2} \mathrm{CsSb}$ compound is along the $\mathrm{X}$-symmetry point. Moreover, the compounds having Cs element exhibit small bandgap as compared to the other compounds having $\mathrm{K}$ and $\mathrm{Rb}$ elements with sodium. The calculated energy gaps along with the available experimental data and the other theoretical results are depicted in table 3. For clearer electronic structure, we have used three schemes namely; WC-GGA, EV-GGA and TB-mBJ for treating the exchange-correlation approximation. Among all the studied compounds, only $\mathrm{Na}_{2} \mathrm{KSb}$ and $\mathrm{K}_{2} \mathrm{CsSb}$ have the relevant previous experimental and theoretical data to be compared with. WC-GGA underestimates, while EV-GGA and mBJ approximations overestimate the experimental results. Having no previous experimental or theoretical data for the other systems, these calculations can serve as reference data for future investigations on these compounds. 

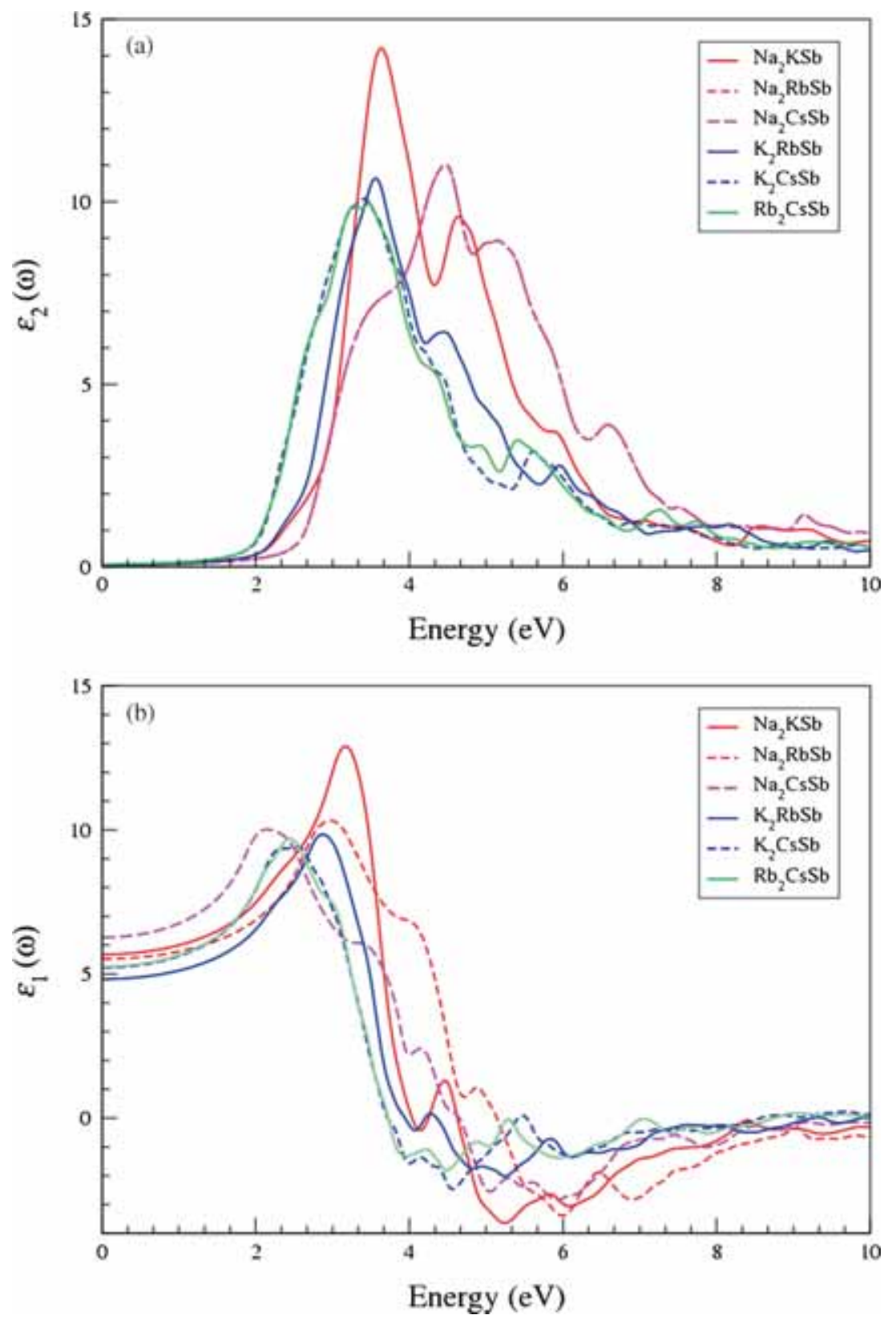

Figure 6. (a) Imaginary part and (b) real part of dielectric function as a function of energy.

Table 4. Present calculated various optical parameters for the understudied compounds.

\begin{tabular}{lccccc}
\hline \multicolumn{5}{c}{ Energy range for } \\
Compound & $\varepsilon_{1}(0)$ & negative $\varepsilon_{1}(\omega)$ & $n(0)$ & $\sigma_{\max }(\omega)$ & $R(0)$ \\
\hline $\mathrm{Na}_{2} \mathrm{KSb}$ & 5.67 & $4.04-11.76$ & 2.38 & $6980(3.68 \mathrm{eV})$ & $16.71 \%$ \\
$\mathrm{Na}_{2} \mathrm{RbSb}$ & 5.52 & $5.15-12.28$ & 2.35 & $6637(4.51 \mathrm{eV})$ & $16.24 \%$ \\
$\mathrm{Na}_{2} \mathrm{CsSb}$ & 6.26 & $4.66-10.24$ & 2.50 & $5313(4.77 \mathrm{eV})$ & $18.41 \%$ \\
$\mathrm{~K}_{2} \mathrm{RbSb}$ & 4.82 & $4.36-9.86$ & 2.19 & $5117(3.60 \mathrm{eV})$ & $14.00 \%$ \\
$\mathrm{~K}_{2} \mathrm{CsSb}$ & 5.22 & $3.74-8.55$ & 2.28 & $4651(3.50 \mathrm{eV})$ & $15.30 \%$ \\
$\mathrm{Rb}_{2} \mathrm{CsSb}$ & 5.25 & $3.74-8.74$ & 2.29 & $4652(3.51 \mathrm{eV})$ & $15.40 \%$ \\
\hline
\end{tabular}


Density of states (DOS) enables one to examine the contribution of different electronic states in the valence and conduction bands to understand the electronic band structure of a material. In addition, DOS also determines various interband and intra-band transitions between quantized electronic levels in valence as well as conduction band to explain optical spectra. The calculated total DOS (TDOS) are shown in figure 4a. Three main bands appear in the energy range of -10 to $10 \mathrm{eV}$. The partial DOS is shown in figure $4 \mathrm{~b}$. The semicore bands of the compounds are formed by the Sb-s state. The valence band is mainly composed of Sb-p state. In the conduction band major contribution is from the $\mathrm{K}, \mathrm{Rb}$, Cs-d and Sb-p states.
The understanding of the charge allocations and chemical bonding among the constituents requires the knowledge of electronic charge distributions. For this purpose, the charge density difference is calculated in (110) plane and depicted in figure 5. From the figures it is seen that the electronic charge distribution is highly localized to the ionic sites, with small distribution between the ions as indicated by the scale. This behaviour indicates the dominant ionic bond character in these compounds. Large charge transfer among the anions and cations can be seen, resulting in well-spaced and spherical shape of the charge distributions in the contour plots. Figure also shows small difference in the ionicity of the understudied compounds. $\mathrm{K}_{2} \mathrm{RbSb}, \mathrm{K}_{2} \mathrm{CsSb}$ and $\mathrm{Rb}_{2} \mathrm{CsSb}$
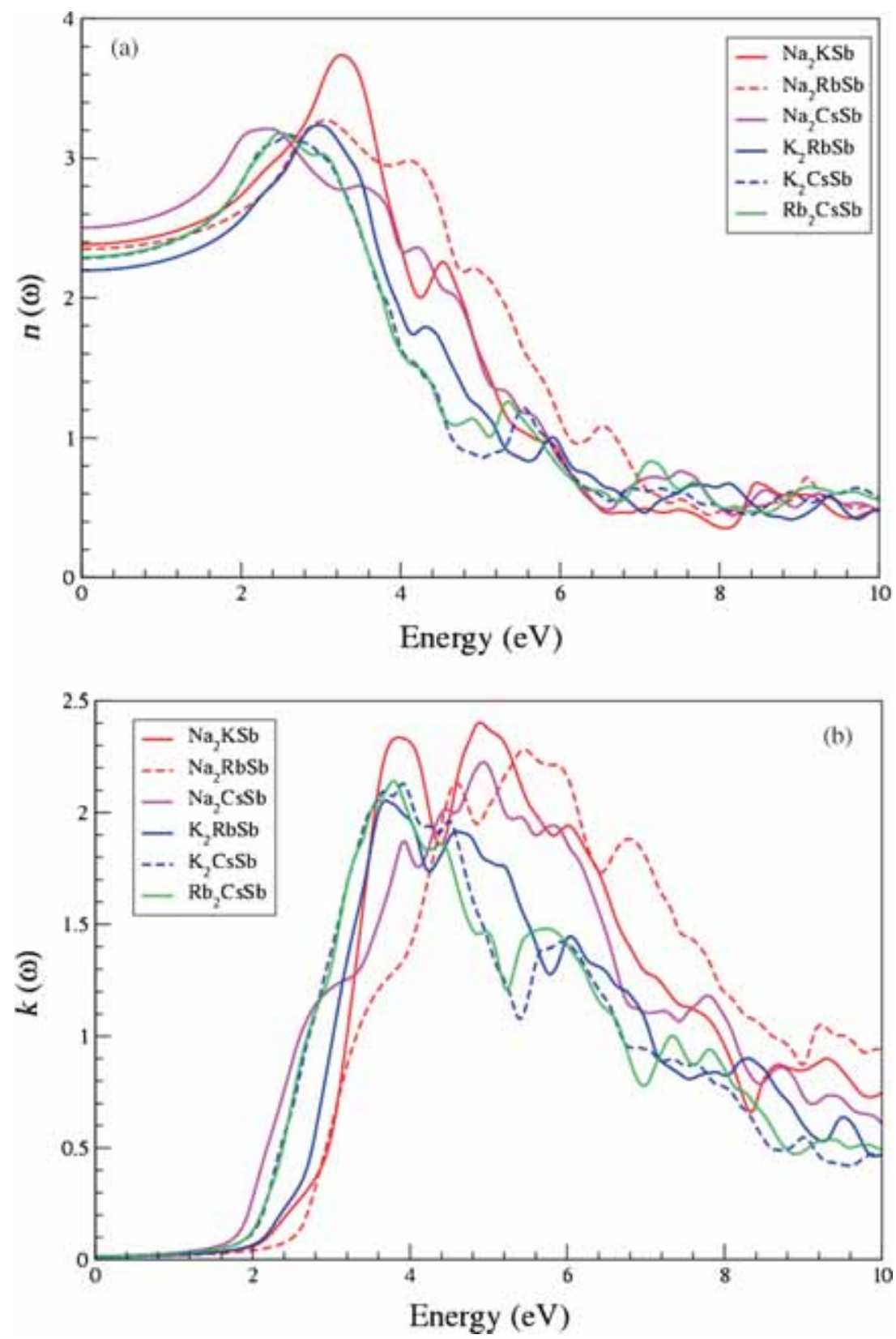

Figure 7. (a) Real part of the refractive index and (b) extinction coefficient as a function of energy. 
can be seen as more ionic in contrast to the sodium-based bi-alkali compounds.

\subsection{Optical properties}

The optical properties of the compounds are estimated from the dispersion relation of the complex dielectric function $\varepsilon(\omega)$. The energy eigenvalues and wavefunctions are the natural output of the band structure calculations, which are needed for the calculation of the dispersion parameter $\varepsilon(\omega)$. The cubic symmetry of these compounds involves only one non-zero component of the dielectric tensor to complete the characterization of their optical properties. The imaginary part of the complex dielectric function $\varepsilon_{2}(\omega)$ is determined by the following relation [35]:

$$
\varepsilon_{2}(\omega)=\frac{8}{2 \pi \omega^{2}} \sum_{n n^{\prime}} \int\left|P_{n n^{\prime}}(k)\right|^{2} \frac{d s_{k}}{\nabla \omega_{n n^{\prime}}(k)},
$$

where the imaginary part is strongly dependent on the joint density of states $\omega_{n n^{\prime}}$ and the momentum matrix element $P_{n n^{\prime}}$. Peaks in the spectra are due to the allowed electronic transitions between the valence and conduction bands. The $\varepsilon_{2}(\omega)$ spectral response is illustrated in figure $6 \mathrm{a}$, which shows transparency of these compounds to the electromagnetic waves until the photon energies reach to their fundamental energy gap. Spectra show three important spectral critical
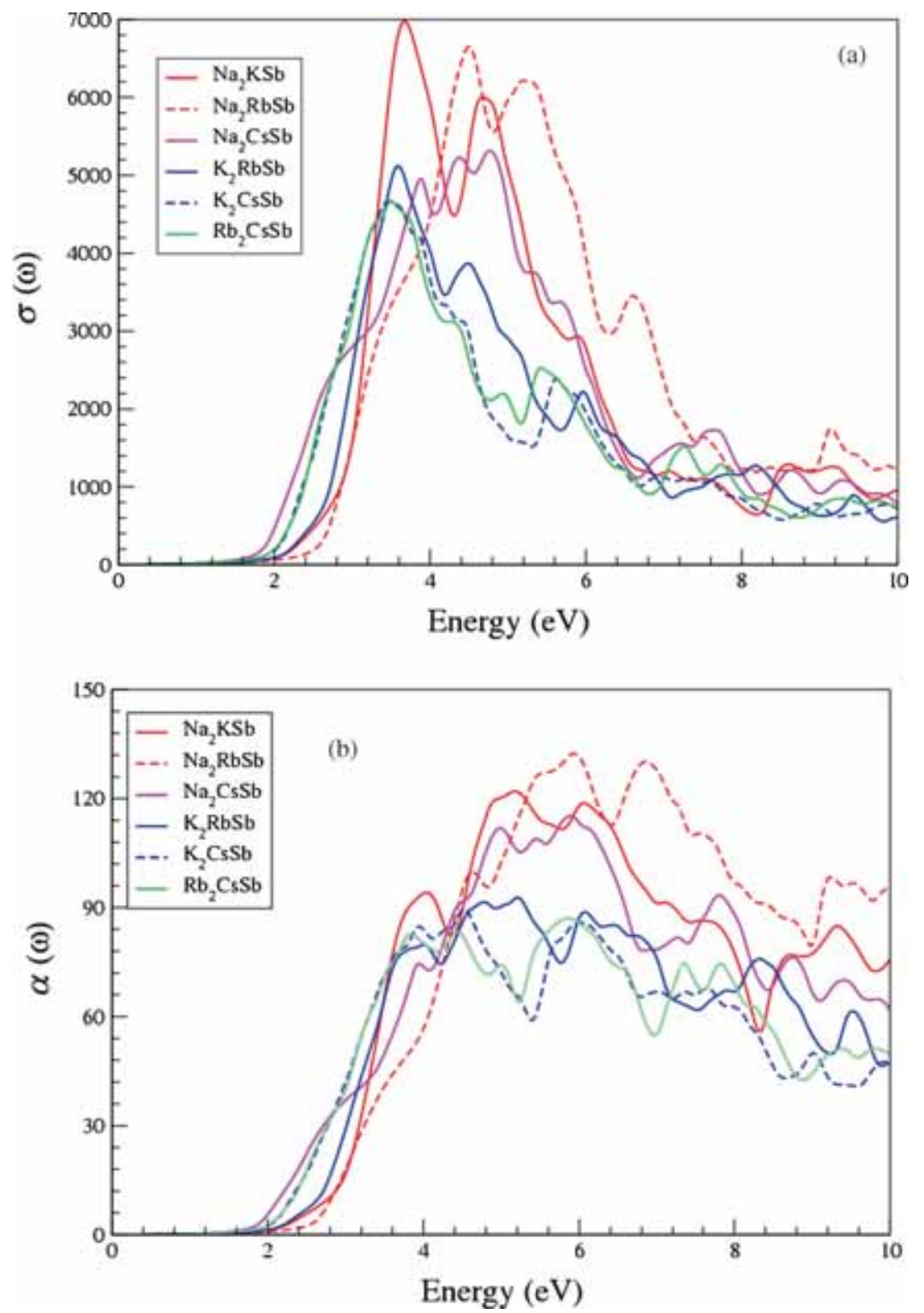

Figure 8. Frequency-dependent (a) optical conductivity and (b) absorption coefficient. 
points in all cases, which related to the direct optical transitions at different symmetry points in the band structure. For the spectra of $\mathrm{Na}_{2} \mathrm{KSb}, \mathrm{K}_{2} \mathrm{RbSb}$ and $\mathrm{K}_{2} \mathrm{CsSb}$ compounds, the fundamental absorption edge along with the next two peaks correspond to the direct optical $\Gamma-\Gamma, X-X$ and $L-L$ transitions. In the case of $\mathrm{Rb}_{2} \mathrm{CsSb}$, the fundamental absorption is due to the direct $X-X$ optical transition, while the next two peaks are due to the direct $\Gamma-\Gamma$ and $K-K$ optical transitions from the valence band maximum to the conduction band minimum. These direct optical transitions are mainly from valence band $\mathrm{Sb}$-p occupied state to the unoccupied d-state of second single cation atom.

The real part $\varepsilon_{1}(\omega)$ can be calculated from the imaginary part using Kramers-Kronig inversion:

$$
\varepsilon_{1}(\omega)=1+\frac{2}{\pi} P \int \frac{\omega^{\prime} \varepsilon_{2}\left(\omega^{\prime}\right)}{\omega^{\prime 2}-\omega^{2}} \mathrm{~d} \omega^{\prime} .
$$

$\varepsilon_{1}(\omega)$ spectra of the herein studied compounds are depicted in figure $6 \mathrm{~b}$, and show no significant interaction up to the incident photon energies less than their energy gap and remain transparent in this energy interval. The limit value of the real part of the complex dielectric function obtained at frequency of irradiations looming up to the zero limit is known as static dielectric constant $\varepsilon_{1}(0)$. According to the Penn model [36], there is an inverse relationship between the minimum energy gap $E_{\mathrm{g}}$ and static dielectric constant $\varepsilon_{1}(0)$, as given by the following relation

$$
\varepsilon_{1}(0) \approx\left(\hbar \omega / E_{\mathrm{g}}\right)^{2},
$$

where $\omega$ is the plasma frequency at which the material's response changes from metallic to dielectric nature. Comparison of the two tables for minimum energy gap and $\varepsilon_{1}(0)$ reveals the inverse relation between these quantities, in accordance to the above model. After the maximum value, the spectra decreases and goes below zero. The negative region corresponds to the attenuation where the maximum reflectivity occurs, which is a common effect due to the increase in the free carrier concentration. Static dielectric constant along with the energy range for the negative values of $\varepsilon_{1}(\omega)$ are given in table 4 .

The dispersion spectra of the real and imaginary parts of the dielectric function are used for calculations of the complex refractive index, optical conductivity, absorption coefficient and reflectivity of the understudied compounds. The real part of the refractive index is shown in figure 7a. The calculated non-zero tensor components of the static refractive indices $n(0)$ are given in table 4 . The static refractive index also varies inversely with the bandgap. The agreement with the Penn model is a consequence of the direct dependence on the static value of $\varepsilon_{1}(\omega)$. The extinction coefficient $\kappa(\omega)$ shown in figure $7 \mathrm{~b}$ illustrates the attenuation of the incident electromagnetic radiations in the energy range from 2.80 to $8 \mathrm{eV}$. The maximum peaks of $\kappa(\omega)$ are located at 24.9, 5.47, 4.94, 3.70, 3.91 and $3.80 \mathrm{eV}$ for $\mathrm{Na}_{2} \mathrm{KSb}, \mathrm{Na}_{2} \mathrm{RbSb}$, $\mathrm{Na}_{2} \mathrm{CsSb}, \mathrm{K}_{2} \mathrm{RbSb}, \mathrm{K}_{2} \mathrm{CsSb}$ and $\mathrm{Rb}_{2} \mathrm{CsSb}$, respectively.

Figure $8 \mathrm{a}$ displays the calculated frequency-dependent optical conductivity $\sigma(\omega)$. Optical conductivity illustrates the conduction of free charge carriers over a defined range of the photon energies. The optical spectra of $\sigma(\omega)$ can be related to the imaginary part of the dielectric function. This is obvious from agreement in the response spectra of $\sigma(\omega)$ and $\varepsilon_{2}(\omega)$. In figure 8b, absorption coefficient $\alpha(\omega)$ spectrum illustrates the interplay between the incident photon energies and their absorption by these materials.

Figure 9 displays the frequency-dependent reflectivity $R(\omega)$ spectra, where the materials exhibit transparency to the frequencies up to their fundamental band edge. The maximum reflectivity peaks are attributed to the increase in the available free charge carriers. This is also evident from

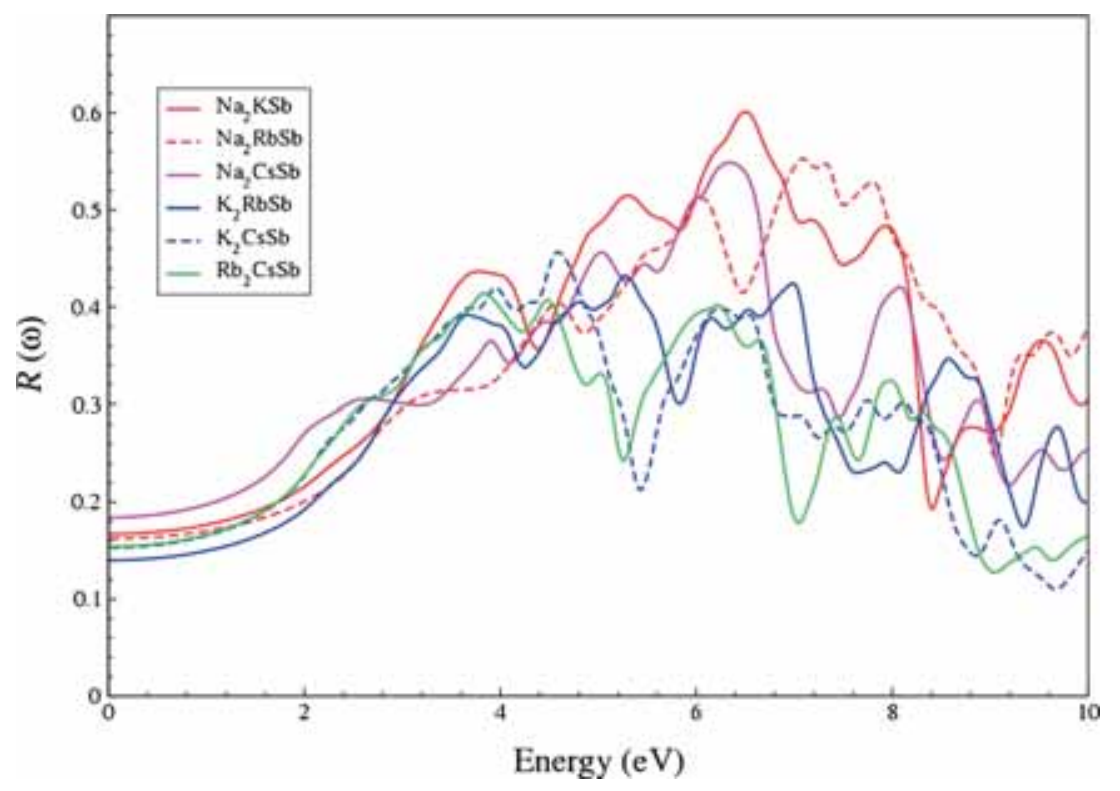

Figure 9. Frequency-dependent reflectivity of the bi-alkali antimonides. 
the agreement of the maximum reflection and the metallic behaviour (negative $\varepsilon_{1}(\omega)$ ) energy ranges. The low frequency reflectivity and maximum optical conductivity along with the optical constants are given in table 4, providing reference data for future purposes.

\section{Conclusions}

The structural parameters, elastic constants, electronic and optical properties of bi-alkali antimonides $\left(\mathrm{Na}_{2} \mathrm{KSb}\right.$, $\mathrm{Na}_{2} \mathrm{RbSb}, \mathrm{Na}_{2} \mathrm{CsSb}, \mathrm{K}_{2} \mathrm{RbSb}, \mathrm{K}_{2} \mathrm{CsSb}$ and $\mathrm{Rb}_{2} \mathrm{CsSb}$ ) were calculated using the FP-LAPW+lo method utilizing WCGGA, EV-GGA and TB-mBJ potentials. Lattice constant increases with increase in the ionic radii of the cation from $\mathrm{Na}$ to $\mathrm{K}$ to $\mathrm{Rb}$. Bulk moduli of the compounds are below $25 \mathrm{GPa}$; therefore, these compounds are not very hard materials. All the compounds are mechanically stable. $\mathrm{Na}_{2} \mathrm{KSb}$, $\mathrm{Na}_{2} \mathrm{CsSb}$ and $\mathrm{K}_{2} \mathrm{RbSb}$ are ductile, while the remaining compounds are brittle. Among the compounds $\mathrm{Na}_{2} \mathrm{KSb}, \mathrm{K}_{2} \mathrm{RbSb}$, $\mathrm{K}_{2} \mathrm{CsSb}$ and $\mathrm{Rb}_{2} \mathrm{CsSb}$ have direct bandgaps; therefore, they are optically active. Chemical bonding among the cations and anions is mainly ionic. The compounds show a high response to the photons in the visible and ultraviolet energy regions. These compounds therefore can be utilized in these energy regions.

\section{Acknowledgements}

We would like to extend our sincere appreciation to the Deanship of Scientific Research at King Saud University for funding this Research Group (No. RG 1435-004).

\section{References}

[1] Ruiz-Osés M, Schubert S, Attenkofer K, Ben-Zvi I, Liang X, Muller E, Padmore H, Rao T, Vecchione T, Wong J Xie J and Smedley J 2014 APL Mater. 2121101

[2] Spicer W E 1958 Phys. Rev. 112114

[3] Taft E and Philipp H R 1959 Phys. Rev. 1151583

[4] Spicer W E 1961 J. Phys. Chem. Solids 22365

[5] Sommer A H 1963 Appl. Phys. Lett. 362

[6] Mc Carroll W H 1965 J. Phys. Chem. Solids 26191

[7] Ebina A and Takahashi T 1973 Phys. Rev. B 74712

[8] Ghosh C and Varma B P 1978 J. Appl. Phys. 494549
[9] Tzolov M B and Iliev M N 1992 Thin Solid films 213 99

[10] Erjavec B 1994 Vacuum 45617

[11] Kong S H, Kinross-Wright J, Nguyen D C and Sheffield R L 1995 Nucl. Instrum. Meth. A 358617

[12] Michelato P 1997 Nucl. Instrum. Meth. A 393455

[13] Ghosh C 1980 Phys. Rev. B 221972

[14] Christensen N E 1985 Phys. Rev. B 32207

[15] Wei S-H and Zunger A 1987 Phys. Rev. B 353952

[16] Ettema A R H F and de Groot R A 1999 J. Phys. Condens. Matter 11759

[17] Ettema A R H F and de Groot R A 2000 Phys. Rev. B 61 10035

[18] Ettema A R H F and de Groot R A 2002 Phys. Rev. B 66 115102

[19] Ettema A R H F 2003 Appl. Phys. Lett. 823988

[20] Kalarasse L, Bennecer B and Kalarasse F 2010 J. Phys. Chem. Sol. 71314

[21] Blaha P, Schwarz K, Madsen G K H, Kvasnicka D and Luitz J 2001 WIEN2k, an augmented plane wave plus local orbitals program for calculating crystal properties, Vienna University of Technology, Austria

[22] Wong K M, Alay-e-Abbas S M, Shaukat A, Fang Y and Lei Y 2013 J. Appl. Phys. 113014304

[23] Wong K M, Alay-e-Abbas S M, Fang Y, Shaukat A and Lei Y 2013 J. Appl. Phys. 114034901

[24] Wu Z and Cohen R E 2006 Phys. Rev. B 73235116

[25] Engel E and Vosko S H 1993 Phys. Rev. B 4713164

[26] Tran F and Blaha P 2009 Phys. Rev. Lett. 102226401

[27] Sajid A, Murtaza G and Reshak A H 2013 Modern Phys. Lett. B 271350061

[28] Birch F 1947 Phys. Rev. 71809

[29] Hill R 1952 Proc. Phys. Soc. Lond. $\mathbf{A 6 5} 349$

[30] Voigt W 1928 Lehrbush der Kristallphysik (Leipzig: Taubner Press)

[31] Reuss A and Angew A 1929 Mater. Phys. 949

[32] Wu Z-J, Zhao E J, Xiang H P, Hao X F, Liu X J and Meng J 2007 Phys. Rev. B 76054115

[33] Peng F, Chen D, Fu H and Cheng X 2009 Phys. Stat. Solid B 24671

[34] Pugh S F 1954 Philos. Mag. 45823

[35] Wooten F 1972 Optical properties of solids (New York: Academic)

[36] Penn D 1962 Phys. Rev. 1282093 